\title{
Análisis de la fertilidad de los suelos de Alto Loica en San Pedro de Melipilla, Región Metropolitana (Chile)
}

\author{
Soil fertility analysis in Alto Loica San Pedro de Melipilla, \\ Metropolitan Region (Chile)
}

\author{
Juan Araya Silva
}

\begin{abstract}
Resumen
En Chile, la erosión es el principal problema ambiental debido a que sobre el $40 \%$ de los suelos en el país está con problemas de erosión, limitando la capacidad de éstos para generar bienes y servicios. En la zona central del país, este fenómeno se intensifica en la Cordillera de la Costa, siendo las comunas rurales las más afectadas, principalmente la comuna de San Pedro de Melipilla, al SW de la Región Metropolitana. Este estudio tiene como objetivo analizar las propiedades físicas y químicas del suelo de Alto Loica, sector rural localizado al sur de la comuna de San Pedro. El estudio se efectuó en 4 microcuencas hidrográficas en Alto Loica, mediante el muestreo de suelos y el análisis de calicatas. Los resultados reflejaron que existen déficit críticos en las concentraciones de micro y macronutrientes, debido a lo cual los suelos presentan baja fertilidad y baja calidad y no son aptos para el desarrollo agropecuario, limitándose su uso solo a aptitud forestal. Por otra parte, esto se puede asociar a malas prácticas agrícolas efectuadas en el pasado, que derivaron en la erosión de los suelos del sector. Este estudio puede contribuir como un marco conceptual y referencial para futuros estudios de restauración y rehabilitación de suelos con el fin de recuperar la propiedad de los suelos de generar bienes y servicios.
\end{abstract}

Palabras clave: suelos, erosión, fertilidad, macronutriente, micronutriente

\begin{abstract}
In Chile, soil erosion is one of the main environmental problems as over $40 \%$ of the country's soils are subjected to erosion, limiting its capacity to generate goods and services. In the central areas of the country, this phenomenon is intensified at the coastal mountain ridge, with rural areas as the most affected. This is the case of the commune of San Pedro de Melipilla, located at the SW of the Metropolitan Region. The aim of this study is to analyze the physical and chemical characteristics of the soil in Alto Loica, which is a rural area located in the southern area of the commune of San Pedro. The study was carried out in four hydrographic micro-basins through the sampling of soils and the analysis of soil pits in Alto Loica. The results show critical deficits in the concentrations of micro and macro nutrients, resulting in low fertility and quality of soils. Thus, the soils of Alto Loica are not apt for agriculture-livestock use, limiting its use to forestry. Moreover, these could be related to poor agricultural practices in the past, which led to soil erosion in the area. Overall, this study could become a conceptual and referential framework for future soil restauration and rehabilitation studies aiming at recovering the soil ability to generate goods and services.
\end{abstract}

Keywords: soil, erosion, fertility, macronutrient, micronutrient

Recibido el 27 de febrero de 2017, aceptado el 15 de mayo de 2017. 


\section{Introducción}

El suelo es un sistema altamente dinámico y está íntimamente relacionado con todos los elementos a los cuales da soporte, por ello se define como un medio biogeoquímico con una alta capacidad de interacción, lo cual es determinante para la actividad agrícola debido a la interacción nutriente-sueloplanta (Hernández, Chailloux, Moreno, Igarza, \& Ojeda, 2014).

En Chile, los suelos carecen de una normativa legal de carácter taxativa debido a la radical liberalización de los mercados chilenos en el siglo pasado. Ello generó un aumento en las externalidades ambientales debido al carácter multisectorial de su uso y regulación (Aguayo, Pauchard, Azócar, \& Parra, 2009) producto de lo cual, las actividades antrópicas (fuertemente marcadas por el aumento de los pasivos ambientales), han ocasionado en forma progresiva cambios físicos y químicos que han degradado en forma progresiva el suelo (Soto, Arriagada, Castro, Maerker, \& Rodolfi, 2011), lo cual, se manifiesta en la pérdida de calidad, decrecimiento de la productividad y una alteración en sus funciones ecológicas (Ghafari et al., 2017).

En Chile se reconoce a la erosión, desde el punto de vista ambiental y socioeconómico, como uno de los problemas ambientales de mayor relevancia a nivel nacional (Meza \& Castro, 2013). A fines de los años setenta, el $36 \%$ de los suelos de Chile continental presentaban problemas de erosión (Bonilla, Reyes, \& Magri, 2010), en la última década del siglo XX, la superficie total de suelos erosionados alcanzó una superficie de 34.480 .800 ha, lo que equivale a un $46 \%$ de los suelos del territorio nacional y cerca del $75 \%$ de los suelos productivos de Chile, de los cuales, el $80,5 \%$ presentaba categorías de erosión clasificadas de moderada a muy severa, que significó que estos suelos habían perdido entre el $40 \%$ y el $100 \%$ de su profundidad total (Francke, 1999). Entre las zonas rurales semiáridas de Chile, ubicadas en la zona central del país (entre la IV Región y la Región Metropolitana), la Cordillera de la Costa representa el sector más afectado por la erosión hídrica, constituyendo éste último el proceso físico de mayor relevancia e incidencia en los procesos erosivos. Todo esto, se debe principalmente al efecto de la tala indiscriminada para el consumo de leña, el sobrepastoreo sin control y la deforestación (entre otros factores), originando que los terrenos pierdan su resistencia a los procesos erosivos causando una disminución en la producción silvoagropecuaria agravando con ello los problemas ambientales e impidiendo el desarrollo sustentable en zonas rurales (Wang, Zheng, Römkens, \& Darboux, 2013). Los problemas ambientales más significativos son la pérdida del potencial agrícola, el aumento de la escorrentía superficial y la disminución de la infiltración, entre otros.

La comuna de San Pedro de Melipilla presenta procesos de erosión hídrica calificados de moderados a muy severos, afectando cerca del $69 \%$ de la superficie comunal. Ubicada al SurEste (SE) de Santiago, San Pedro es la única comuna en la Región Metropolitana que aún es $100 \%$ rural y la más vulnerable del secano interior en la zona central de Chile. Sus habitantes son principalmente medianos y pequeños propietarios, herederos de la reforma agraria entre 1960 y 1973, con un alto nivel de pobreza, debido en parte, al uso irracional de los recursos naturales de la zona (Francke, Yoma, Carnieletto, Rivera, \& Nakagawa, 2008), por lo que la deforestación excesiva para su uso como combustible y producción de carbón (sumado a una ganadería y agricultura intensiva donde primó el monocultivo de trigo), produjo la deforestación de cientos de hectáreas, perdiendo con ello el suelo la capa vegetal que lo protegía contra la lluvia y las escorrentías erosionando los suelos de la zona. Debido a lo anterior, la Corporación Nacional Forestal (CONAF) efectuó entre 1993 y 1998 en conjunto con 
la Agencia de Cooperación Internacional de Japón (JICA) un proyecto de restauración de cuencas hidrográficas en el sector de Alto Loica con el objetivo de restaurar suelos degradados disminuyendo con ello la superficie erosionada mediante la implementación de obras de restauración hidrológico-forestales; todo ello con el propósito de recuperar los suelos y potenciar su capacidad productiva.

Lo anterior tenía como fin la transferencia de tecnología a los habitantes del sector para disminuir la pobreza rural. Ello denota la importancia de recuperar suelos degradados y establecer a futuro planes de sustentabilidad que complementen la presión de los asentamientos humanos con los beneficios otorgados por el recurso suelo. El presente estudio tiene como objetivo analizar las propiedades físicas y químicas del suelo de Alto Loica, para que puedan constituirse como base, marco conceptual y marco referencial para futuros planes de rehabilitación y sustentabilidad de suelos en el sector, con el fin de recuperar los servicios ecosistémicos del suelo y restablecer la propiedad de los suelos y de generar bienes y servicios para los asentamientos humanos disminuyendo la pobreza rural de la comuna.

\section{Material y métodos}

\section{Sitio de estudio}

La comuna de San Pedro se localiza en el extremo SW de la Región Metropolitana (figura 1) en la zona semiárida del secano costero interior de la zona central de Chile en la cuenca del rio Maipo y tiene una superficie aproximada de 80.000 ha, de las cuales el $99,94 \%$ es rural y solo el $0,06 \%$ es de carácter rural urbano (Aguirre, 2009). Esta zona se inserta en la hoya del estero Yali, el cual se ubica entre los sectores bajos de los ríos Maipo y Rapel, extendiéndose en dirección E-NW con una longitud aproximada de casi $50 \mathrm{Km}$, desde su naciente a 360 m.s.n.m. hasta desembocar en el mar. El área donde se desarrolla el estudio corresponde a la localidad de Alto Loica en el extremo sur de la comuna de San Pedro entre los paralelos $33^{\circ} 46^{\prime}$ y $34^{\circ}$ $04^{\prime}$ Latitud Sur y los entre los meridianos $71^{\circ} 12^{\prime}$ y $71^{\circ} 42^{\prime}$ Longitud Oeste, a $38 \mathrm{Km}$. de distancia del océano Pacífico y a 120 Km. de Santiago. En San Pedro las series de los suelos predominantes son Cauquenes (CQ), Estancilla (STC), Lingolingo (LL) y Lo Vásquez (LVZ) (Francke et al., 2008). Los suelos de las cuencas Alto Loica se definen como la serie de suelos Lo Vásquez, de textura franco arcillo arenosa, de profundidad media y muy susceptibles a la erosión. La capacidad de uso se define entre VI y VII de A.P.F (Aptitud Preferentemente Forestal), lo que implica que constituyen suelos severamente afectados por la erosión, y se clasifican como suelos forestales.

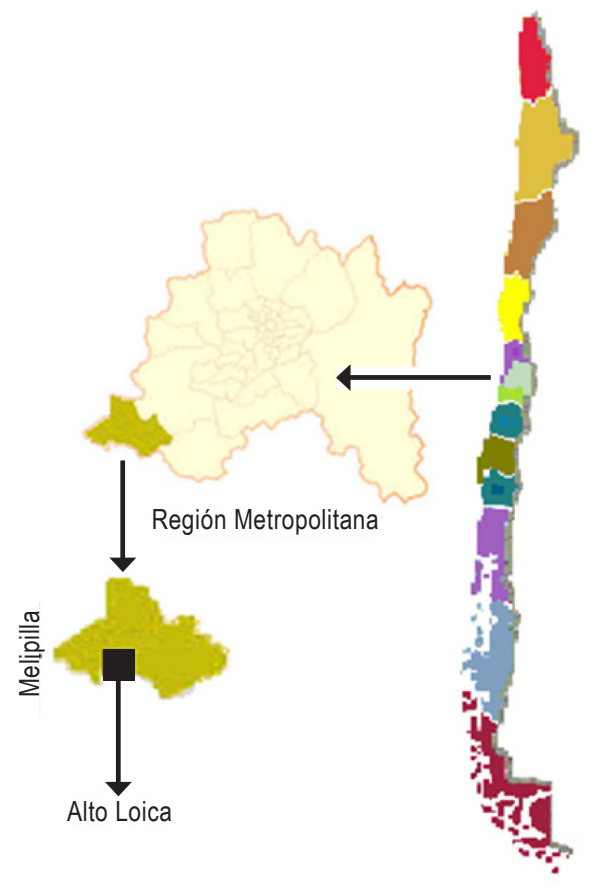

Figura 1. Ubicación de la zona de estudio (Alto Loica) al SW de la Región Metropolitana. Fuente: Elaboración propia (2017)

Figure 1.Location of the study area (Alto Loica), Southwest of the Metropolitan Region. Source:

Own elaboration (2017) 


\section{Metodología de muestreo y análisis}

El proyecto CONAF-JICA en Alto loica contempló la delimitación y estudio de 4 microcuencas claramente definidas $y$ georeferenciadas (figura 2), cuyos suelos pertenecen a la serie Lo Vásquez con una superficie que fluctúa entre las 14 y 34 ha. En 3 de ellas (MC1, MC2 y MC3) se instalaron dispositivos de control de erosión, mientras que la microcuenca 4 se uso como microcuenca testigo, no instalándose en ella ninguna obra hidrológica forestal.

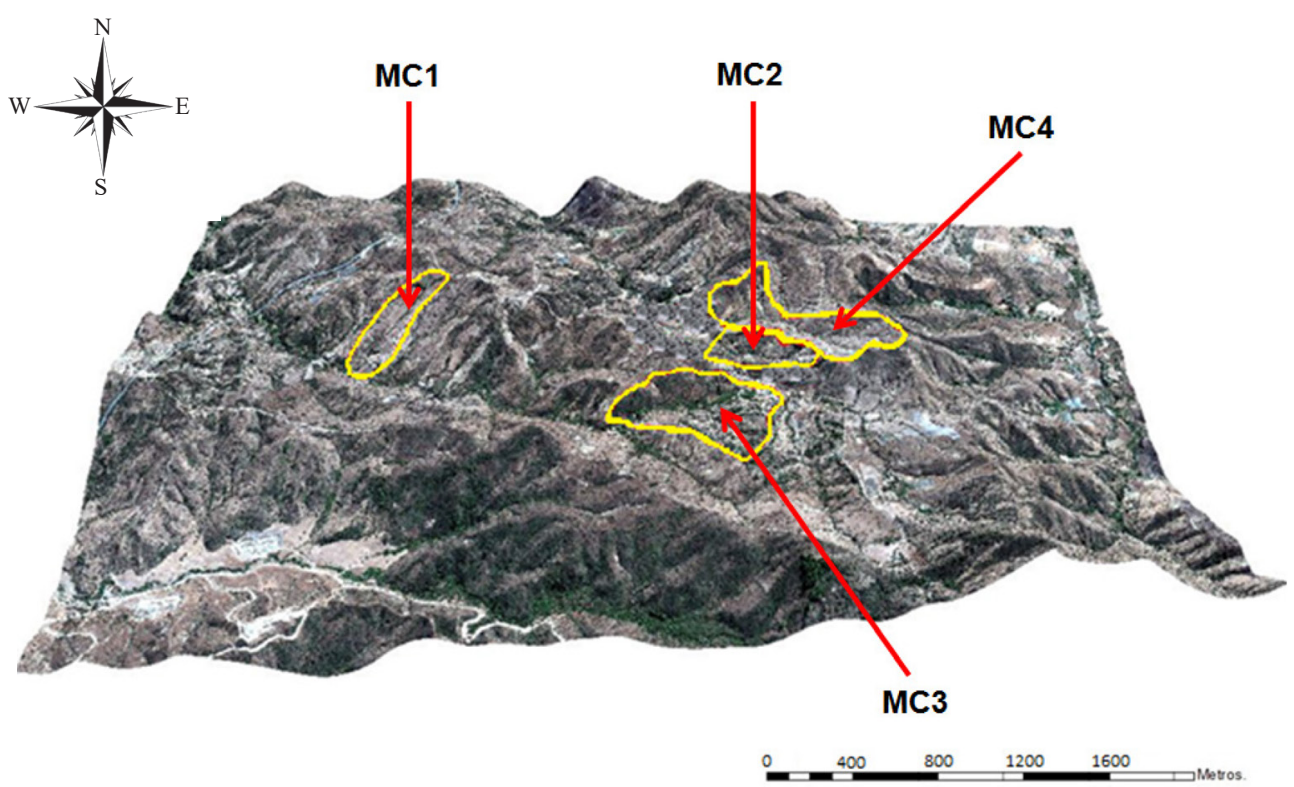

Figura 2. Localización espacial de las microcuencas (MC) de trabajo en Alto Loica. Fuente: Elaboración propia (2017)

Figure 2. Space location of the microbasins of work (MB) in Alto Loica. Source: Own elaboration (2017)

Análisis físico. Para el análisis físico se excavaron un total de 12 calicatas en la microcuencas de trabajo bajo el siguiente protocolo: una en la zona alta, media y baja de las microcuencas respectivamente. Las dimensiones de las calicatas fueron de 0,8 por $1,2 \mathrm{mt}$ con una profundidad de $1,2 \mathrm{mt}$, esto según el procedimiento descrito por Schlatter, Grez, \& Gerding (2003).

Análisis químico. Para este análisis se determinaron los puntos de muestreo (tabla
1) en función de las respuestas de las obras hidrológica-forestales a los procesos de restauración en los últimos 20 años. Por ello, 5 de los 8 puntos de muestreo (figura 3) se obtuvieron en la microcuenca 2 y 1 en cada microcuenca restante (MC1, MC2 y MC4). Para el muestreo se usó un bastón pedológico a una profundidad de 50 centímetros para la obtención del horizonte mineral. Se tomaron 10 submuestras de un punto para homogeneizarlas y obtener una muestra compuesta y representativa del sector. 
Tabla 1

Geolocalización y caracterización de la posición fisiográfica y altitud de los puntos de muestreo en las microcuencas de trabajo. Fuente: Elaboración propia (2017)

Table 1

Geolocation and characterization of the physiographic position and altitude of the sample spots in the microbasins of work. Source: Own elaboration (2017)

\begin{tabular}{llll}
\hline Punto & Coordenadas UTM & Posición fisiográfica & Altitud (msnm) \\
\cline { 2 - 4 } 1.1 & $0268643 \mathrm{mE} / 6236432 \mathrm{mS}$ & Lomaje & 296 \\
2.1 & $0270145 \mathrm{mE} / 6236015 \mathrm{mS}$ & Lomaje & 286 \\
2.2 & $0270087 \mathrm{mE} / 6235906 \mathrm{mS}$ & Lomaje & 300 \\
2.3 & $0270002 \mathrm{mE} / 6235844 \mathrm{mS}$ & Terraza & 320 \\
2.4 & $0270031 \mathrm{mE} / 6235815 \mathrm{mS}$ & Terraza & 320 \\
2.5 & $0270098 \mathrm{mE} / 6235840 \mathrm{mS}$ & Terraza & 300 \\
3.1 & $0270128 \mathrm{mE} / 6235347 \mathrm{mS}$ & Colina & 294 \\
4.1 & $0270523 \mathrm{mE} / 6235947 \mathrm{mS}$ & Lomaje & 317 \\
\hline
\end{tabular}

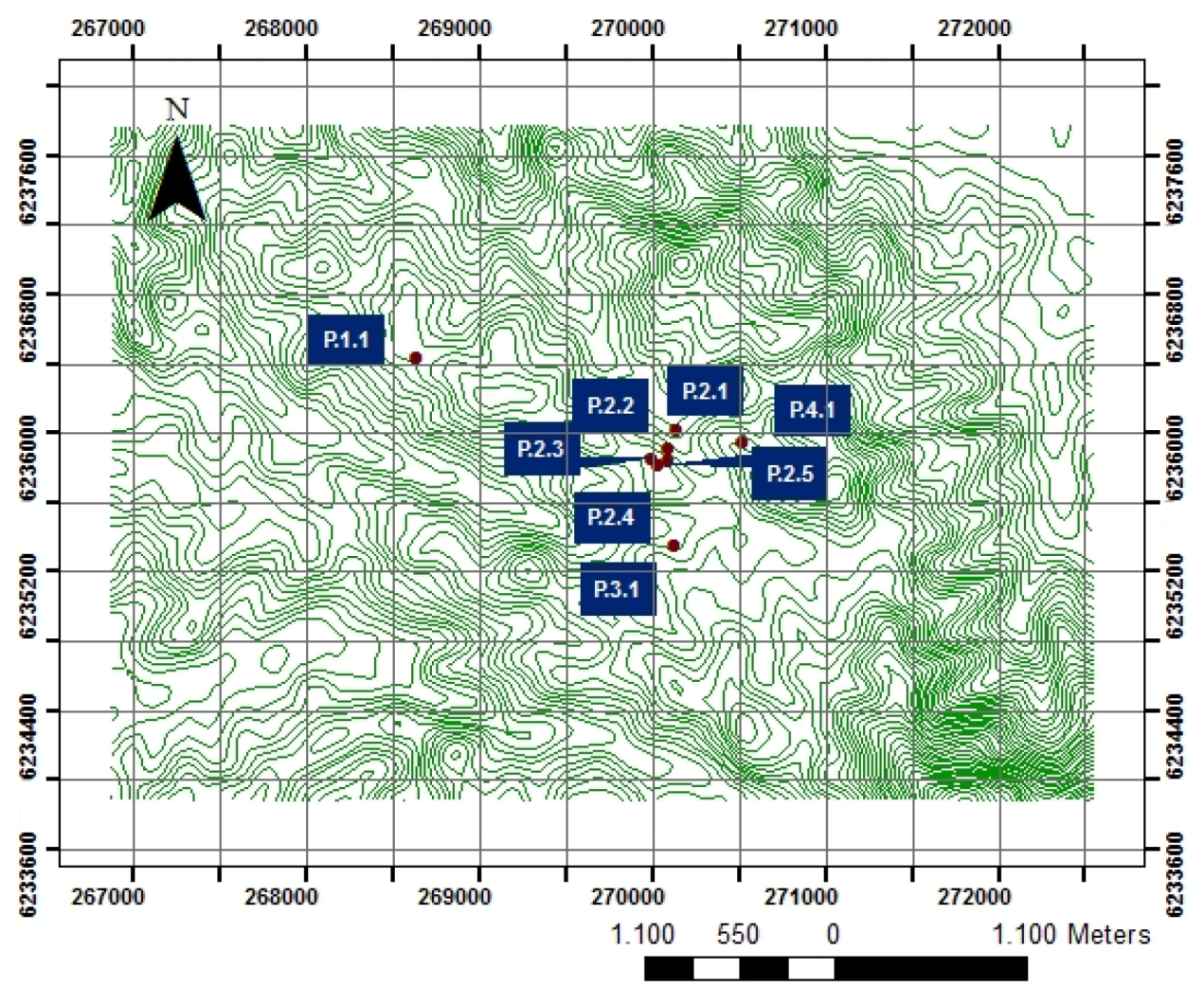

Figura 3. Grid de puntos de muestreo. Fuente: Elaboración propia (2017)

Figure 3. Grid of sampling points. Source: Own elaboration (2017) 
Cada submuestra tenía el mismo volumen de extracción eliminándose los desechos que pudiesen estar contenidos en ella; una vez completadas las submuestras se homogeneizaron en un recipiente previamente determinado para ello sin elementos ajenos ni tratamiento alguno que interfiriera con los análisis. De estas muestras compuestas se constituyó una muestra única de $0,7 \mathrm{Kg}$ para su análisis en laboratorio. Las muestras fueron colocadas en bolsas plásticas resistentes al transporte previamente rotuladas con las características del sitio de recolección, no introduciéndose (según protocolo) ningún elemento ajeno al interior de las bolsas. Las muestras fueron enviadas al laboratorio certificado según norma ISO 17025, INIA La Platina, para los análisis que comprendieron; macronutrientes (N, P, K); Materia orgánica (MO); Capacidad de intercambio catiónico (CIC); $\mathrm{pH}$ y micronutrientes ( $\mathrm{Fe}, \mathrm{Zn}, \mathrm{Mn}, \mathrm{Ca}, \mathrm{Cu}$ y $\mathrm{B}$ ). Conjuntamente a lo anterior, los análisis contemplaban la capacidad de campo, punto de marchitez permanente y densidad.

\section{Resultados}

\section{Aspectos físicos}

El suelo tiene una profundidad que fluctúa entre los 40 y 70 centímetros, se puede distinguir 5 horizontes con una transición en profundidades de $11 \mathrm{~cm}, 28$ $\mathrm{cm}, 43 \mathrm{~cm}$, y $68 \mathrm{~cm}$. En el perfil se puede manifestar el proceso de iluviación de arcilla por la presencia de la estructura prismática que tiene un límite lineal con el horizonte C (figura 4). La clase textural es franco arenosa (sL) en la parte media y más arenosa en las partes superior e inferior con abundante grava de cuarzo. El horizonte $\mathrm{C}$ es muy denso y duro. La consistencia muy dura es producto de la gran sequía estival. Los colores son pardos en todos los horizontes, salvo el tercero que es más rojizo. Los procesos en este suelo son oxidación por el cambio anual del equilibrio hídrico.

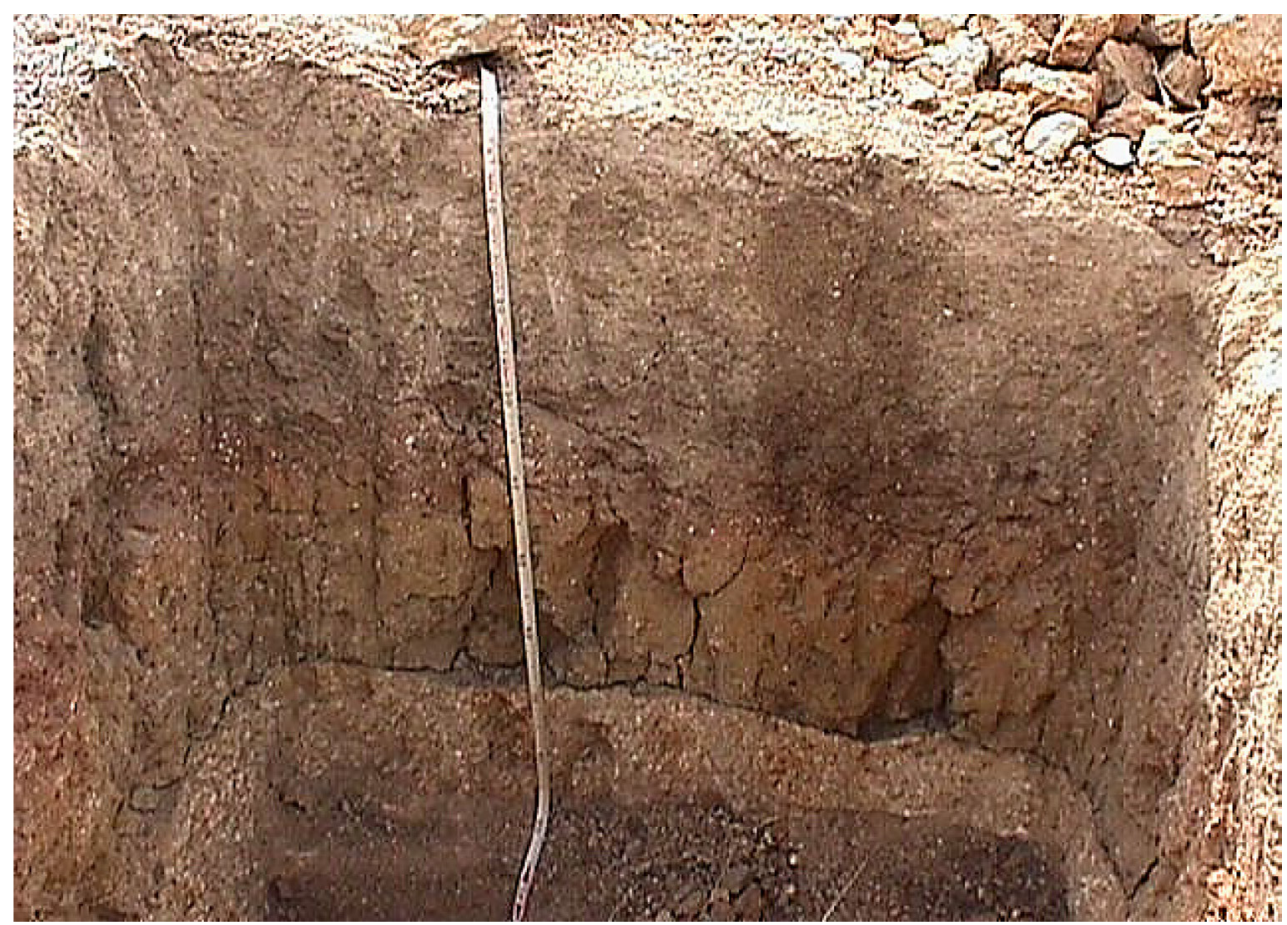

Figura 4. Suelo con clase textural franco arenosa. Fuente: Elaboración propia (2017)

Figure 4. Soil with sandy loam textural class. Source: Own elaboration (2017) 
El primer horizonte presenta óxidos de hierro de color anaranjados. La parte bajo la capa de grava y piedras presenta óxidos de manganeso, de color negros. La clase textural varía entre franco arenosa $(\mathrm{sL})$, arena franca (IS) y limo arenoso (sU) con una profundidad que no supera los 50 centímetros en el horizonte $\mathrm{B}$ y una pedregocidad que fluctúa entre el $2 \%$ en los horizontes superiores y un $15 \%$ en los horizontes subsuperficiales y una densidad aparente entre 1,30 gr/cc y
$2,00 \mathrm{gr} / \mathrm{cc}$ respectivamente. Respecto al contenido de agua, existen variaciones según la textura (siendo mayor en el caso de los suelos arcillosos), en el caso de Alto Loica la capacidad de campo (CC) fluctúa entre un $24 \%$ y un $31 \%$; el punto de marchitez permanente entre el $7 \%$ y $17 \%$; la capacidad de agua aprovechable exhibe fluctuaciones entre un $14 \%$ y $18 \%$. Finalmente la capacidad de agua aprovechable (CAA) encuentra valores entre 25,2 y $30,6 \mathrm{~mm}$.

Tabla 2

Matriz de correlación de los factores hidrológicos del suelo. Fuente: Elaboración propia (2017)

Table 2

Correlation matrix of the hydrological factors of the soil. Source: Own elaboration (2017)

\begin{tabular}{lllll}
\hline & CC & PMP & CAA (\%) & CAA (mm) \\
\cline { 2 - 5 } CAA $(\mathbf{m m})$ & -0.87758533 & -0.94800662 & 0.99715504 & \\
CAA $(\%)$ & -0.85434186 & -0.93286524 & \\
PMP & 0.98419917 & & \\
CC & & & \\
\hline
\end{tabular}

Los datos anteriores presentan un alto grado de correlación indicado en la tabla 2. El agua que migra hacia los horizontes subsuperficiales es utilizada en forma efectiva por las plantas hasta un nivel que está determinado por la porosidad y las condiciones climáticas; por ejemplo, en los horizontes superiores el suelo almacena aproximadamente solo un $13,72 \%$ de precipitación como agua aprovechable para las plantas; este valor presenta un alto grado de correlación con la capacidad de campo y el punto de marchitez permanente. Esto explica en parte la sequedad del sector y a su vez condiciona la relación $\mathrm{pH}-\mathrm{CIC}$, la cual no muestra correlación debido a que la sequedad del suelo no facilita el ingreso o salida de los cationes desde los sitios de intercambio catiónico de la arcilla.

\section{Aspectos químicos}

Los análisis muestran que el pH fluctúa entre los 4.5 - 5.4 en el horizonte superficial y entre los 5.6 - 6.7 en el horizonte más profundo (mineral), por lo que son ácidos en la superficie y básicos en los horizontes más internos. La oscilación del $\mathrm{pH}$ no es muy pronunciada lo que indica que el proceso de basicidad en las zonas bajas no está muy pronunciada ni avanzada. En los horizontes superficiales hay materia orgánica en descomposición (como por ejemplo restos de pinus radiata $\mathrm{y}$ pinus pinea) proveniente de restos vegetales, los cuales, al encontrarse en descomposición presentan un bajo $\mathrm{pH}$ debido a la formación de ácidos fúlvicos y la alta capacidad de intercambio catiónico. La tabla 3 muestra que el $\mathrm{pH}$ superficial del suelo es ligeramente ácido, lo que se explica por la presencia de materia orgánica de naturaleza ácida, además, la alta presencia de nitrógeno, contribuye a la adsorción de hidrogeniones lo que impide una disminución progresiva del $\mathrm{pH}$. 
En los horizontes internos del suelo, la cantidad de materia orgánica disminuye más de un $90 \%$, lo que genera a su vez una disminución de los coloides y de micronutrientes, por lo que el $\mathrm{pH}$ se debe casi en su totalidad a los minerales originarios del suelo. La alta compactación del suelo contribuye a su vez a una menor infiltración de agua y por lo tanto no hay una acidificación de suelo al no existir intercambio de los $\mathrm{H}^{+}$del agua por $\mathrm{Ca}^{++}$ por lo que no se satura. De la misma forma, el $\mathrm{pH}$ condiciona las especies iónicas y la movilidad de estos elementos, ya que, la migración o inmovilidad de ellos, depende del $\mathrm{pH}$ presente en el suelo.

Tabla 3

Aspectos químicos y concentraciones de macronutrientes en el suelo de Alto Loica. Fuente:

Elaboración propia (2017)

Table 3

Chemical aspects and concentration of macronutrients in the soil of Alto del Loica. Source: Own elaboration (2017)

\begin{tabular}{llllllll}
\hline Punto & $\begin{array}{l}\mathbf{C I C} \\
\mathbf{c m o l}+\mathbf{K g}\end{array}$ & $\mathbf{p H}$ & $\begin{array}{l}\mathbf{C .} \mathbf{E} . \\
\mathbf{m S} / \mathbf{c m}\end{array}$ & $\begin{array}{l}\mathbf{M} . \mathbf{O} . \\
\mathbf{1}\end{array}$ & $\begin{array}{l}\mathbf{N} \\
\mathbf{p p m}\end{array}$ & $\begin{array}{l}\mathbf{P} \\
\mathbf{p p m}\end{array}$ & $\begin{array}{l}\mathbf{K} \\
\mathbf{p p m}\end{array}$ \\
\cline { 2 - 7 } 2.1 & 15.20 & 6.00 & 0.45 & 2.3451 & 5 & 5 & 75 \\
2.2 & 16.5 & 5.86 & 0.27 & 1.23140 & 10 & 8 & 38 \\
2.3 & 12.3 & 5.68 & 0.30 & 2.02600 & 3 & 6 & 80 \\
2.4 & 26.4 & 6.11 & 0.32 & 1.49300 & 15 & 4 & 58 \\
2.5 & 14.6 & 6.52 & 0.29 & 2.19800 & 4 & 4 & 75 \\
3.1 & 8.5 & 6.01 & 0.48 & 2.79300 & 7 & 4 & 169 \\
4.1 & 18.3 & 6.53 & 0.63 & 2.81800 & 6 & 4 & 111 \\
\hline
\end{tabular}

La tabla 3 indica que la materia orgánica es abundante en el mantillo pero disminuye en forma drástica en los horizontes más profundos a niveles tales que no resulta significativa en la retención de agua aprovechable; la alta compactación, el clima de secano interior y la alteración de la microbiota producto de la erosión (Araya \& Atero, 2016) disminuyen la tasa de mineralización en el suelo; esto implica que en los horizontes más profundos disminuyen la CIC, la biodisponibilidad, la mantención del pH y el ciclo de nutrientes.

Respecto del N, K y el P, no solo son necesarios para la nutrición y desarrollo de los vegetales, sino que además contribuyen a otorgar resistencia a las plantas contra la bajas temperaturas y por ende, dan mayor resistencia al frío (De Souza et al., 2011). Esta característica es importante en las zonas de secano interior, ya que este sector 
presenta estaciones muy marcadas con altas oscilaciones térmicas y bajas temperaturas en las noches durante el invierno.

La tabla 4 muestra las concentraciones de los micronutrientes en los que cabe destacar al nitrógeno que juega un papel preponderante en los ciclos ecosistémicos naturales (De Souza et al., 2011); la falta de este elemento ocasiona la reducción en el crecimiento foliar y clorosis uniforme de hojas que es típico de los eucaliptus de Alto Loica. El P se encuentra entre un $80 \%$ a $90 \%$ de déficit en relación a la marca de clase del rango óptimo de concentración (43 ppm); en el K en cambio, solo una muestra supera el rango normal (169 ppm), lo cual no es significativo comparado con el resto de las muestras, las cuales, son un $27 \%$ a $75 \%$ más bajo en relación a un nivel óptimo.

Tabla 4

Concentraciones de micronutrientes en el suelo de Alto Loica. Fuente: Elaboración propia (2017)

Table 4

Concentrations of micronutrients in the soil of Alto Loica. Source: Own elaboration (2017)

\begin{tabular}{lllllllll}
\hline Punto & $\begin{array}{l}\mathbf{F e} \\
\mathbf{p p m}\end{array}$ & $\begin{array}{l}\mathbf{Z n} \\
\mathbf{p p m}\end{array}$ & $\begin{array}{l}\mathbf{M n} \\
\mathbf{p p m}\end{array}$ & $\begin{array}{l}\mathbf{C u} \\
\mathbf{p p m}\end{array}$ & $\begin{array}{l}\mathbf{C a} \\
\mathbf{c m o l} / \mathbf{k g}\end{array}$ & $\begin{array}{l}\mathbf{C} \text {. Total } \\
\mathbf{( \% )}\end{array}$ & $\begin{array}{l}\text { Relación } \\
\mathbf{C} / \mathbf{N}\end{array}$ & $\begin{array}{l}\mathbf{B} \\
\mathbf{p p m}\end{array}$ \\
\cline { 2 - 8 } 1.1 & 80.787 & 0.56 & 56.98 & 1.29 & 5.34 & 1.38 & 14.37 & 0.32 \\
2.1 & 150.40 & 1.16 & 62.80 & 1.70 & 4.92 & 0.714 & 10.82 & 0.15 \\
2.2 & 39.98 & 0.28 & 51.60 & 1.08 & 3.57 & 1.175 & 26.98 & 0.33 \\
2.3 & 26.34 & 0.22 & 47.60 & 1.16 & 5.32 & 0.866 & 18.83 & 0.23 \\
2.4 & 7.24 & 0.20 & 17.78 & 0.14 & 11.28 & 1.275 & 16.78 & 0.22 \\
2.5 & 17.86 & 0.42 & 19.38 & 0.48 & 7.28 & 0.8008 & 14.05 & 0.20 \\
3.1 & 29.94 & 0.30 & 51.74 & 1.00 & 2.10 & 1.620 & 11.41 & 0.41 \\
\hline 4.1 & 20.76 & 0.70 & 31.80 & 0.86 & 6.93 & 1.635 & 17.77 & 0.27 \\
\hline
\end{tabular}

Algunos micronutrientes como el Mn, Zn, $\mathrm{Fe}$ y $\mathrm{Cu}$ disminuyen su disponibilidad a medida que el pH aumenta (Ginocchio \& Narváez, 2002). Un pH ligeramente acido $(6,5)$ conlleva a la liberación de $\mathrm{Fe}^{+3}$ desde ciertas especies minerales (Ginocchio \& Narváez, 2002) lo que provoca una coloración ocre que es típica en algunos horizontes de los suelos de Alto Loica, aunque, la mayor concentración de este elemento está presente en los horizontes más profundos. La figura 5 indica que la semejanza varía entre 83,1605 y 25,0349 según análisis dendrográfico; esto indica que la variabilidad química del suelo de Alto Loica se mantiene en determinadas concentraciones, con lo cual se infiere que los niveles de los macro y micronutrientes se deben básicamente a la composición del material de origen. 


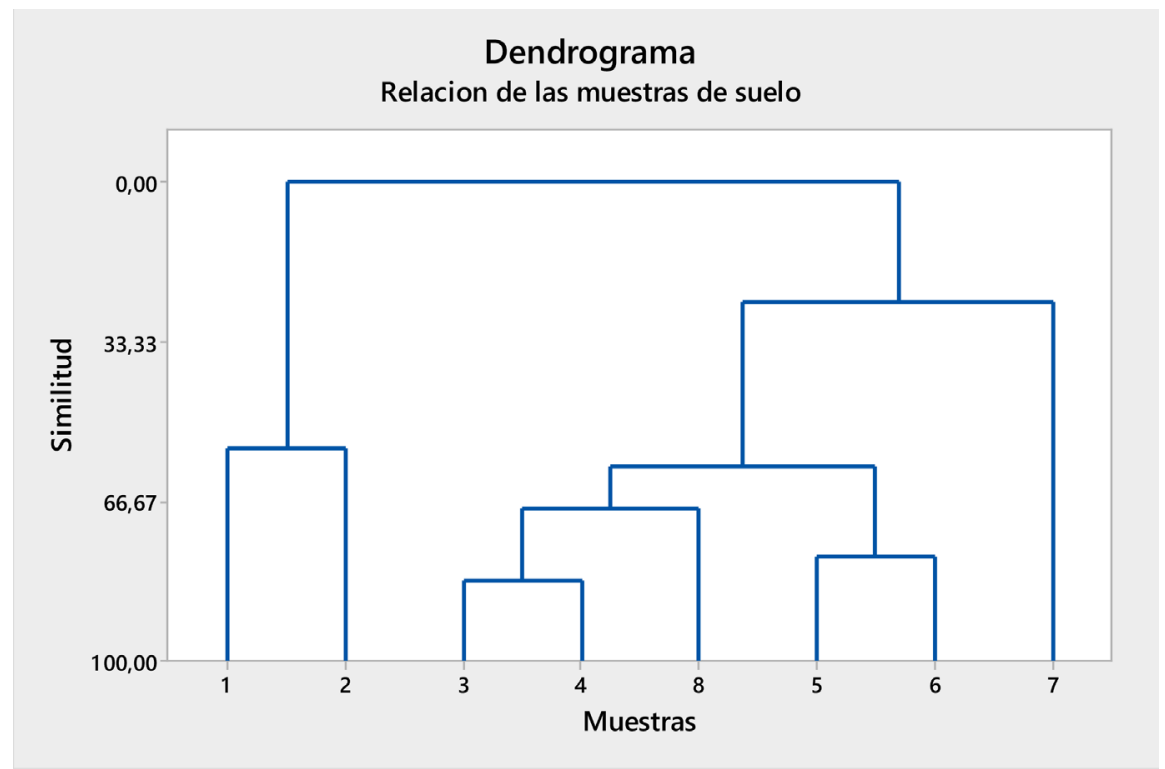

Figura 5. Dendograma de relación de las muestras del suelo. Fuente: Elaboración propia (2017)

Figure 5. Dendogram of relation of soil samples. Source: Own elaboration (2017)

El B es requerido para el óptimo crecimiento de la vegetación por lo que es un oligoelemento esencial, aunque, en cantidades excesivas se vuelve tóxico (Rummukainen et al., 2013). Sin embargo, los niveles de este elemento en los suelos de Alto Loica es muy bajo sobretodo en el horizonte mineral $(0.15 \mathrm{ppm}-0.41 \mathrm{ppm})$, cuya concentración es muy baja con relación al rango normal óptimo que fluctúa entre los 1ppm - 5 ppm (Malavé, Carrero, Lemus, \& García, 2009). Esto último es perceptible en las hojas de Eucaliptus globulus y Eucaliptus camaldulenses donde las hojas amarillas presentan motas o sequedad en los tejidos. Estos síntomas avanzan desde la punta a lo largo de los bordes y hacia el centro de la hoja entre los nervios (Rummukainen et al., 2013). Esto último que es muy notorio en los eucaliptus, ya que es uno de los arboles más representativos de las microcuencas, del área de estudios por ser una de las especies más usadas para la reforestación debido a su resistencia al estrés hídrico (Oyarzún, Frêne,
Lacrampe, Huber, \& Hervé, 2011). El B es un elemento esencial para el desarrollo de este árbol por lo que su carencia (o niveles muy por debajo del rango de $1 \mathrm{ppm}$ a $5 \mathrm{ppm}$ ) afecta su desarrollo arbóreo reflejándose en una disminución en el diámetro del tronco y alturas muy por debajo de lo habitual. Esto último es visible en los eucaliptus de la microcuenca 2, los cuales, se secan aproximadamente a los 15 años. El análisis de componentes principales del suelo de Alto Loica (figura 6) indica una asociación entre capacidad de intercambio catiónico y $\mathrm{pH}$ en conjunto con los niveles de calcio. De la misma forma, la conductividad eléctrica se mantiene constante en función de la materia orgánica. El Zn es un elemento soluble en los suelos cuyo comportamiento está fuertemente influido por el $\mathrm{pH}$ : la adsorción de $\mathrm{Zn}$ baja a pH menor a 7, lo que implica que la adsorción de este elemento es menor en los horizontes superficiales del suelo y a $\mathrm{pH}$ más alto forma complejos orgánicos solubles, la cual debería ser la 
forma en la cual este elemento se encuentra en los horizontes más profundos del suelo. Este elemento es importante para la vegetación forestal, ya que participa en procesos metabólicos como la respiración y la síntesis de clorofila y proteínas (Woch, Stefanowicz, \& Stanek, 2017); por ello la carencia o bajos niveles de este elemento afecta fuertemente el metabolismo vegetal. Sin embargo, en los suelos de Alto Loica los valores superan en muchos estratos las $5 \mathrm{ppm}$, lo que se considera aceptable debido a que el rango óptimo para el crecimiento y desarrollo vegetal es de 0,5 ppm - 1 ppm (Puga, Mello, Mattiuz, Do vale, \& Fonseca, 2013).

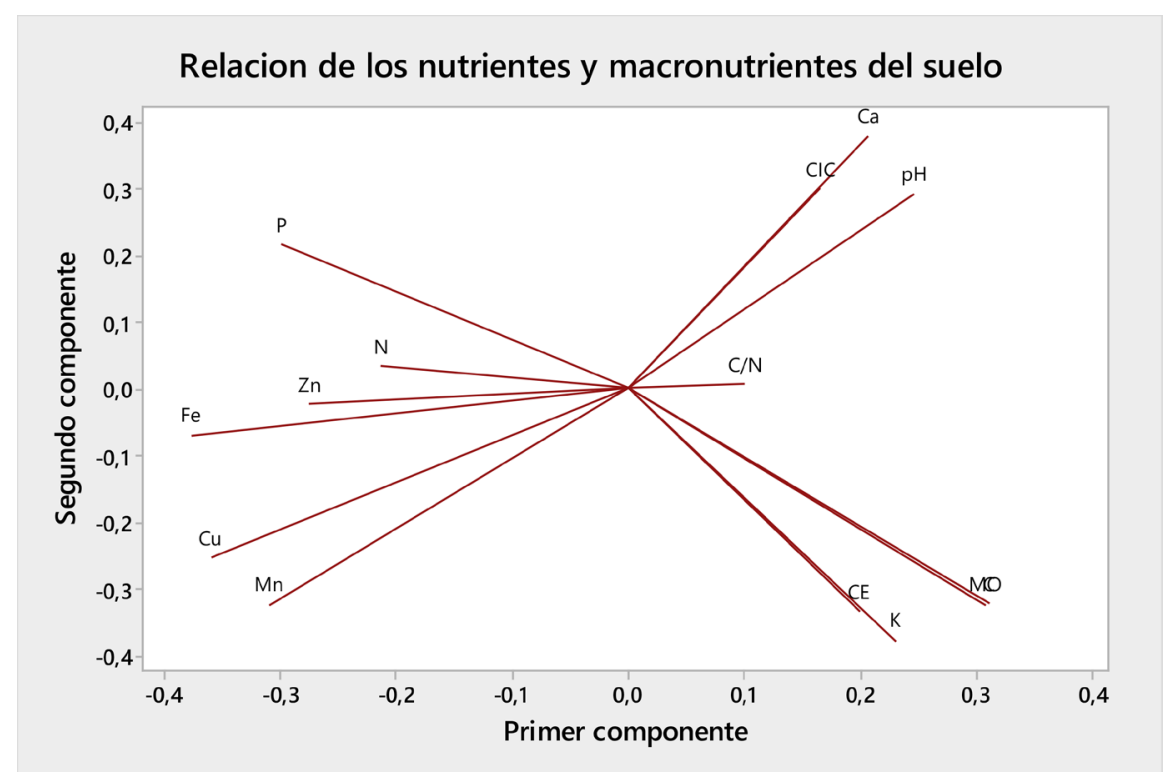

Figura 6. Análisis multivariado de los componentes principales del suelo. Fuente: Elaboración propia (2017)

Figure 6. Multivariate analysis of the main soil components. Source: Own elaboration (2017)

El Mn es el elemento presente en mayor concentración en los suelos de Alto Loica, fluctuando entre los 469 ppm - 632 ppm en los horizontes superficiales y los $17 \mathrm{ppm}$ $62 \mathrm{ppm}$ en el horizonte mineral. Se presenta como $\mathrm{Mn} 2+$ en la solución del suelo y en el complejo de cambio por lo que esta debiera ser la forma dominante en los estratos superiores del suelo debido a la presencia de coloides que aumentan los sitios de intercambio, además es posible encontrarlo como MnO2 (Roca, Susana, \& Bech, 2007).

\section{Discusión}

Los procesos erosivos en Chile son consecuencia de prácticas agrícolas ineficientes, ganadería intensiva y deforestación, hechos que se intensificaron durante la segunda mitad del siglo XX (Francke et al., 2008). Estos procesos tuvieron un impacto más intenso en el sector de Alto Loica en San Pedro de Melipilla debido a la inexistencia de procesos de depositación, así como por 
las condiciones climáticas que obedecen a un clima mediterráneo de secano interior de estaciones marcadas con lluvias que no superan los $300 \mathrm{~mm}$ que solo se restringen a la temporada invernal.

Los suelos de Alto Loica son de clase textural franco arenoso, arena franca y arcillo arenosa, debido a que la Cordillera de la Costa está compuesta básicamente por rocas extrusivas graníticas y metamórficas como material original. Su textura reduce la capacidad de retención de agua en un 50\% en comparación a un suelo arcilloso, por ejemplo, los suelos del piedemonte de la zona sureste de Santiago son arcillosos con remanentes de bosques esclerófilo en las zonas altas, condición clave para amortiguar los procesos erosivos y para su uso como suelos agrícolas.

La profundidad del suelo es mayor en las zonas bajas de las microcuencas y menor en las zonas altas, esto implica una pérdida de suelo por efecto de la escorrentía superficial en las zonas altas de las microcuencas generando la depositación en las zonas bajas. Esto ha dado lugar a suelos muy delgados a moderadamente profundos $(40 \mathrm{~cm}$ a 70 $\mathrm{cm})$ con una pedregocidad en promedio moderada de características áridas y secas en las zonas más profundas. Su densidad aparente es muy alta (sobre $2 \mathrm{gr} / \mathrm{cc}$ ), debido a los procesos erosivos y la influencia climática lo cual afecta su capacidad de retención de agua sobre todo en la temporada de noviembre a marzo debido a las altas temperaturas lo que produce un aumento de la evaporación en el suelo aumentando la compactación. Los horizontes superiores tienen abundante grava de cuarzo lo que es característico de los suelos de la Cordillera de la costa de la zona central de Chile con una muy fuerte erosión de manto o zanjas que es típica de los suelos residuales graníticos. Los horizontes subsuperficiales son más densos y compactos, también con abundante grava de cuarzo y descansan en materiales granitoídeos altamente intemperizados. Hay una abundante presencia de cuarzo en todos los perfiles de las microcuencas, además de óxidos de hierro en forma de manchas en el horizonte inferior.

La humedad es muy baja (CAA 15\% - 18\%) debido al porcentaje de agua no aprovechable por las plantas producto de la presencia de arena; aunque la capacidad de campo supera el $30 \%$ en las zonas bajas de las microcuencas es insuficiente para el establecimiento de flora arbustiva, por lo tanto, las características del suelo solo lo hacen aprovechable como suelo forestal.

El análisis químico de los suelos indica bajas concentraciones de micro y macronutrientes en relación a los niveles óptimos necesarios para una alta producción agrícola. El suelo de Alto loica se caracteriza por tener $\mathrm{pH}$ cercano a neutro en las zonas bajas, lo cual, contribuye positivamente a las propiedades físicas del suelo; esto además impide que la estructura del suelo se vuelva inestable o que la arcilla se disperse, además el $\mathrm{pH}$ en los suelos en estudio guarda una relación directa con la $\mathrm{CIC}$, así como con la biodisponibilidad de minerales (Ginocchio \& Narváez, 2002). En los horizontes superiores el $\mathrm{pH}$ es menor $(5,8)$ debido a la presencia de materia orgánica, sin embargo en los horizontes inferiores es mayor $(6,8)$ debido principalmente a los minerales y la baja presencia de agua debido a la compactación del suelo y la baja infiltración (Oyarzún et al., 2011).

De la misma forma, la materia orgánica en los horizontes superiores favorece la mayor presencia de minerales; aunque su concentración disminuye con la profundidad así como su solubilidad debido a la escasa presencia de agua en los horizontes inferiores. En el caso del $\mathrm{N}$, por encontrarse en mayor proporción en los horizontes superiores, la mayor parte de él es arrastrado por la escorrentía superficial debido a su solubilidad y a la escasa vegetación. En el caso del B, sus niveles no sobrepasan la $1 \mathrm{ppm}$; lo cual no suple la cantidad suficiente para el normal 
desarrollo de las plantas; hecho que es visible por la baja biodiversidad presente en las microcuencas (Puga et al., 2013).

A diferencia de los suelos de la zona sur del país, los suelos de Alto Loica presentan niveles de macro y micronutrientes muy por debajo de las concentraciones necesarias para el desarrollo agrícola, debido a que estos compuestos provienen del material de origen, por tanto la importancia de los resultados implican que los suelos son de aptitud forestal y el agotamiento de su capacidad para la generación de bienes y servicios significa la implementación de numerosas obras de restauración hidrológica-forestal además de planes de gestión ambiental que rehabiliten y restauren las zonas degradadas que permitan y mejoren las condiciones para el asentamiento humano.

\section{Conclusiones}

El estado actual de los suelos de Alto Loica es consecuencia de años de sobreexplotación agrícola y ganadera que se efectuaron sin control y sin planes de manejo que dada las condición geomorfológica de la zona eran vitales para la sustentabilidad de los suelos. Lo anterior se debe a que los micro y macronutrientes son aportados básicamente por el material de origen lo que presenta una limitante en comparación a las cuencas cuyos afluentes provienen de la Cordillera de los Andes.

Los suelos de Alto Loica son básicamente suelos de aptitud forestal, lo cual se explica por los remanentes de bosque esclerófilo típico de la zona central con clima mediterráneo con estaciones marcadas y altas oscilaciones térmicas en el secano interior.

Las características físicas y químicas de los suelos resultan vitales para determinar los parámetros agrícolas y su capacidad para la producción de bienes y servicios. Por tanto, estas características son claves para los futuros planes de sustentabilidad aplicables en la zona principalmente por los pequeños y medianos propietarios con el fin de aumentar la producción agrícola y disminuir los niveles de pobreza.

\section{Agradecimientos}

Facultad de Geografía Física de la Universidad de Barcelona, España

Programa de restauración de cuencas hidrográficas de la zona semiárida de Chile de la Corporación Nacional Forestal, CONAF

\section{Referencias}

Aguayo, M., Pauchard, A., Azócar, G., \& Parra, O. (2009). Cambio del uso del suelo en el centro sur de Chile a fines del siglo $\mathrm{XX}$ : Entendiendo la dinámica espacial y temporal del paisaje. Revista chilena de historia natural, 82(3), 361-374. https://doi. org/10.4067/s0716-078x2009000300004

Aguirre, M. (2009). Atlas universal Antártica Chile y sus regiones. Santiago de Chile: Editorial Antártica.

Araya, J. \& Atero, R. (2016). Changes in the soil microbian variability by alteration of native forest. Journal of Technological Possibilism, 6(9): 10-25.

Bonilla, C., Reyes, J., \& Magri, A. (2010). Estimación de la erosión hídrica empleando la ecuación universal de pérdida de suelo revisada (RUSLE) y SIG en Chile central. Chilean journal of agricultural research, 70(1), 159-169. https://doi.org/10.4067/ s0718-58392010000100017

De Souza, V., Caron, B., Schmidt, D., Behling, A., Bamberg, R., \& Vian, A. (2011). Resistance of arboreal species submitted to extreme frost in different agroforestry systems. Ciência Rural, 41(6), 972-977. https://doi.org/10.1590/s0103-847 82011005000073

Francke, S. (1999). Manejo del suelo posible para el desarrollo forestal del semiárido chileno. (Documento Técnico $\mathrm{N}^{\circ}$ 127). Santiago de Chile: CONAF. 
Francke, S., Yoma, R., Carnieletto, C., Rivera, H., \& Nakagawa, M. (2008). Informe técnico de la contribución de Conaf a la estrategia nacional de cuencas. Santiago de Chile: CONAF.

Ghafari, H., Gorji, M., Arabkhedri, M., Roshani, G., Heidari, A., \& Akhavan, S. (2017). Identification and prioritization of critical erosion areas based on onsite and offsite effects. Catena, 156, 1-9. https://doi. org/10.1016/j.catena.2017.03.014

Ginocchio, R. \& Narváez, J. (2002). Importancia de la forma química y de la matriz del sustrato en la toxicidad por cobre en Noticastrum sericeum (Less.) Less. ex Phil. Revista chilena de historia natural, 75(3), 603-612. https://doi.org/10.4067/s07 $16-078 \times 2002000300011$

Hernández, M., Chailloux, M., Moreno, V., Igarza, A., \& Ojeda, A. (2014). Niveles referenciales de nutrientes en la solución del suelo para el diagnóstico nutricional en el cultivo protegido del tomate. Idesia (Arica), 32(2), 79-88. https://doi.org/10.4067/s0718 $-34292014000200011$

Malavé, A., Carrero, P., Lemus, M., \& García, M. (2009). Boron content in coffee plantations (Coffea arabica 1.) in two coffee producing locations of Venezuela. Idesia (Arica), 27(1), 7-12. https://doi.org/ 10.4067/s0718-34292009000100002

Meza, M.\&Castro, C.(2013). Susceptibilidad erosiva asociada al proceso de reconversión agrícola productiva, cuenca semiárida de Quillota. V Región de Valparaíso, Chile. Idesia (Arica), 31(4), 43-52. https://doi. org/10.4067/s0718-34292013000400006

Oyarzún, C., Frêne, C., Lacrampe, G., Huber, A., \& Hervé, P. (2011). Propiedades hidrológicas del suelo y exportación de sedimentos en dos microcuencas de la Cordillera de la Costa en el sur de Chile con diferente cobertura vegetal. Bosque
(Valdivia), 32(1), 10-19. https://doi.org/ 10.4067/s0717-92002011000100002

Puga, A., Mello, R., Mattiuz, B., Do vale, D., \& Fonseca, I. (2013). Efecto de diferentes métodos de aplicación y dosis de zinc sobre la composición química de los granos de maíz y sorgo, cultivados en un suelo Oxisol. Ciencia e investigación agraria, 40(1), 97108. https://doi.org/10.4067/s0718-162020 13000100008

Roca, N., Susana, M., \& Bech, J. (2007). Disponibilidad de cobre, hierro, manganeso, zinc en suelos del NO argentino. Ciencia del suelo, 25(1), 31-42.

Rummukainen, A., Julkunen-Tiitto, R., Ryyppö, A., Kaunisto, S., Kilpeläinen, J., \& Lehto, T. (2013). Long-term effects of boron and copper on phenolics and monoterpenes in Scots pine (Pinus sylvestris L.) needles. Plant and Soil, 373, 485-499. https://doi. org/10.1007/s11104-013-1817-7

Schlatter, J. Grez, R., \& Gerding, V. (2003). Manual para el reconocimiento de suelos. Valdivia: Instituto de Silvicultura, Facultad de Ciencias Forestales, Universidad Austral de Chile.

Soto, M., Arriagada, J., Castro, C., Maerker, M., \& Rodolfi, G. (2011). Relación entre el cambio de uso del suelo en la cuenca del Aconcagua y su litoral arenoso correlativo: Chile central. Revista de geografia Norte Grande, 50, 187 - 202. https://doi. org/10.4067/s0718-34022011000300011

Wang, B., Zheng, F., Römkens, M., \& Darboux, F. (2013). Soil erodibility for water erosion: A perspective and Chinese experiences. Geomorphology, 187, 1-10. https://doi.org/10.1016/j.geomorph.2013.01.018

Woch, M., Stefanowicz, A., \& Stanek, M. (2017). Waste heaps left by historical $\mathrm{Zn}-\mathrm{Pb}$ ore mining are hotspots of species diversity of beech forest understory vegetation. Science of The Total Environment, 599-600, 32-41. https://doi.org/10.1016/j. scitotenv.2017.04.197 